Maria das Dores Nunes ${ }^{1}$ Alberto Madeiro ${ }^{2}$ Debora Diniz ${ }^{3}$

\section{Histórias de aborto provocado entre adolescentes em Teresina, Piauí, Brasil}

\author{
Histories of induced abortion among adolescents \\ from Teresina in the State of Piauí, Brazil
}

${ }^{1}$ Faculdade de Saúde, Ciências Humanas e Tecnológicas do Piauí. R. Vitorino Orthiges Fernandes 6123, Uruguai. 64.057-100 Teresina PI. m.dnunes@hotmail.com ${ }^{2}$ Núcleo de Pesquisa e Extensão em Saúde da Mulher, Universidade Estadual do Piauí. ${ }^{3}$ Programa de PósGraduação em Política Social, Universidade de Brasília.

\begin{abstract}
This paper analyses the methods, techniques and support networks taken by adolescents to perform illegal abortions. It is a descriptive and cross-sectional study involving interviews with 30 adolescents who had been hospitalized for uterine curettage in two public hospitals in Teresina between June and November 2011. Informed consent was given orally, and the interviews were recorded after the confirmation of the induced abortion. The adolescents were between 14 and 17 years old, single, and predominantly lived with their parents in urban areas, had little schooling and recorded a gestational age of 12 weeks. Between 3 and 6 tablets of Cytotec were taken orally and/or vaginally by 28 (94\%) adolescents, and they were rushed to the hospital due to severe cramping, vaginal bleeding or both. They either bought Cytotec alone (43\%, 13), or with the help of a friend or partner $(40 \%, 12)$. Cytotec was sold to them in ordinary pharmacies, by the owner $(45 \%, 13)$ or clerk $(55 \%, 55)$, who provided instructions for use. They went to the hospital with their mother $(40 \%, 12)$ or a girl friend $(30 \%, 9)$. Three (10\%) adolescents developed serious complications. The study revealed that Cytotec is the main method used to perform illegal abortions among adolescents.
\end{abstract}

Key words Adolescence, Induced abortion, Cytotec, Reproductive health, Uterine curettage
Resumo Este artigo analisa os métodos, os percursos e as redes de apoio utilizados por adolescentes para o aborto clandestino. O estudo, transversal e descritivo, entrevistou 30 adolescentes internadas após a curetagem uterina por aborto em dois hospitais públicos de Teresina, de junho a novembro de 2011. O consentimento livre e esclarecido foi oral e as entrevistas foram gravadas após a confirmação do aborto induzido. Majoritariamente, as adolescentes tinham entre 14 e 17 anos, eram solteiras, urbanas, moravam com os pais, tinham baixa escolaridade e registravam idade gestacional de 12 semanas. O Cytotec foi usado isoladamente por 28 (94\%) adolescentes - de 3 a 6 comprimidos, vaginal e/ou oralmente, que procuraram o hospital após sangramento vaginal e/ou cólicas intensas. Elas compraram o Cytotec sozinhas $(43 \%, 13)$ ou com ajuda de amigo ou companheiro (40\%, 12), em farmácias comuns. O medicamento foi vendido pelo proprietário $(45 \%, 13)$ ou pelo balconista $(55 \%, 16)$, que deu as orientações de uso. $O$ apoio para as adolescentes irem ao hospital foi dado pela mãe $(40 \%, 12)$ ou por amiga $(30 \%, 9)$. Houve 3 (10\%) complicações graves, resultando em internação de até 20 dias. O estudo demonstra a predominância do Cytotec como método abortivo entre adolescentes.

Palavras-chave Adolescência, Aborto clandestino, Cytotec, Saúde reprodutiva, Curetagem uterina 


\section{Introdução}

A gravidez na adolescência é uma questão que esteve na pauta da pesquisa acadêmica nas duas últimas décadas. No Brasil, assim como em outros países, houve aumento da proporção de nascimentos em mulheres com menos de 20 anos, ao contrário do decréscimo observado nas demais faixas etárias ${ }^{1,2}$. Dados do Ministério da Saúde demonstram que de 1980 até 2004 ocorreu elevação de 15\% no total de gestações de 10 a 19 anos. Além disso, o parto vaginal tem sido a primeira causa de internação de jovens entre 10 e 14 anos pelo Sistema Único de Saúde em todos os estados do país ${ }^{3,4}$. Há evidências de que a gravidez na adolescência é mais comum na parcela negra da população, predomina na zona rural e, como consequência, ocasiona aumento da taxa de evasão escolar ${ }^{5,6}$.

Entre as adolescentes sujeitas à gestação não planejada, a opção pelo aborto provocado é elevada. Nos países onde o aborto é legalizado, a taxa de interrupção da gravidez entre adolescentes diminuiu no período entre 1995 e $2003^{7}$. Por outro lado, 1 a cada 8 mortes entre mulheres adolescentes no continente africano tem como causa o aborto clandestino, que é proibido na maioria dos países ${ }^{8}$. Em 2008, estimou-se que 41\% dos abortos inseguros em países em desenvolvimento foram feitos entre mulheres de 15 a 24 anos. Desse total, 16\% ocorreram entre adolescentes de 15 a 19 anos na América Latina e no Caribe 9 .

Há poucos dados sobre a magnitude do aborto provocado no Brasil, principalmente em decorrência do contexto restritivo legal. Um levantamento por amostragem aleatória realizado com 2.002 mulheres urbanas em 2010, através da combinação do método da urna e de questionário aplicado por entrevistadoras, evidenciou que $15 \%$ das mulheres entre 18 e 39 anos já realizaram pelo menos um aborto na vida, sendo que $55 \%$ delas necessitaram de internação hospitalar por complicações ${ }^{10}$. Desde a década de 1990 houve redução das complicações graves por aborto induzido e, consequentemente, da mortalidade materna por essa causa ${ }^{11}$. Parte da redução das complicações do aborto inseguro decorreu da introdução do misoprostol (princípio ativo do Cytotec) como substância abortiva no país, que passou a ser rápida e amplamente utilizado pelas mulheres com essa finalidade ${ }^{12}$.

Os estudos brasileiros sobre aborto clandestino entre adolescentes são ainda mais escassos do que entre outras idades. Em geral, a exigência de autorização de um responsável dificulta a realização de pesquisas dessa natureza. Para transpor essa dificuldade, pesquisadores têm lançado mão do resgate da memória sobre as experiências reprodutivas na juventude. Um estudo de caráter qualiquantitativo sobre gravidez e adolescência, realizado com 4.643 jovens entre 18 e 24 anos em três capitais brasileiras (Porto Alegre, Salvador e Rio de Janeiro), demonstrou que o aborto foi relatado por $16,7 \%$ das mulheres como o desfecho da primeira gravidez ${ }^{13}$. A etapa qualitativa dessa pesquisa entrevistou 123 jovens e constatou que, ao descobrir a gravidez não prevista, a maioria das mulheres $(70 \%)$ pensou, tentou ou fez o aborto ${ }^{14}$.

Em outras regiões do país, percentuais semelhantes de aborto provocado entre adolescentes foram descritos. Em 2011, 26,7\% de 2.592 adolescentes de 12 a 19 anos disseram ter induzido o aborto, por meio de entrevistas estruturadas, em Maceió (AL) ${ }^{15}$. Na mesma cidade, em 2012, foi realizado estudo com 201 adolescentes submetidas à curetagem uterina pós-aborto. Através da utilização de critérios indiretos para classificar a prática do aborto, os pesquisadores consideraram que 164 adolescentes se encontravam na categoria de aborto certamente provocado. Dessas, $89 \%$ usaram misoprostol isolado $(20 \%)$ ou combinado $(77 \%)$, 5\% tiveram necessidade de transfusão sanguínea e $93 \%$ tinham idade gestacional acima de 13 semanas ${ }^{16}$.

Os estudos com evidência sobre aborto e adolescentes no Brasil ainda apresentam perguntas não esclarecidas, principalmente em regiões específicas do país. Pouco se sabe, por exemplo, se as práticas abortivas são as mesmas adotadas por mulheres de outras faixas etárias, sobre quem vende e quem compra o misoprostol, sobre como as adolescentes o usam, que sintomas levam à procura pelo hospital e quem as orienta sobre como usá-lo. Além disso, não se conhece quais pessoas auxiliam a adolescente no itinerário da prática abortiva. Este artigo descreve a questão do aborto provocado entre 30 adolescentes internadas para realização de curetagem uterina em Teresina, a quarta capital do país com pior Índice de Desenvolvimento Humano (IDH), onde não existem dados consolidados sobre o tema. O objetivo principal é caracterizar os métodos, o percurso e as redes de apoio das adolescentes para a interrupção da gestação.

\section{Metodologia}

Trata-se de estudo descritivo com delineamento transversal, realizado entre junho e novembro de 2011, na cidade de Teresina (PI). A pesquisa foi 
conduzida em dois hospitais públicos onde há maior parcela de atendimento à mulher no ciclo gravídico-puerperal do município e de regiões vizinhas. No ano de 2010, os dois hospitais concentraram cerca de $80 \%$ das curetagens uterinas realizadas em adolescentes de 10 a 19 anos na capital.

Durante o período de estudo, foram internadas 131 adolescentes de 10 a 19 anos para realização de curetagem uterina por aborto incompleto nos dois hospitais. A primeira autora desta pesquisa, também médica do serviço, identificava as jovens internadas na enfermaria e, após breve exposição, convidava-as para conversar em sala reservada. Nesse momento, havia maior detalhamento do estudo e, em caso de concordância, a entrevista era iniciada. Não houve recusas nas abordagens. Na conversa inicial com a adolescente, nem sempre era possível distinguir se o aborto havia sido espontâneo ou induzido, mas somente foram incluídos no estudo relatos das adolescentes que confirmaram ter induzido o aborto. Trata-se de uma amostra de conveniência, sem seleção prévia das participantes.

As entrevistas foram guiadas por roteiro semiestruturado que explorou três questões centrais: práticas, percursos e redes de apoio. As práticas de aborto correspondiam aos métodos utilizados pelas adolescentes para indução do aborto, tais como medicamentos, chás ou ervas, curiosas ou clínicas. Como as adolescentes fizeram referência ao Cytotec como método abortivo, esse artigo optará pela menção a esse nome comercial, apesar de seu registro ter sido suspenso pela Anvisa em 2011. Atualmente há somente um único medicamento à base de misoprostol (Prostokos) comercializado no Brasil, com uso restrito em ambiente hospitalar ${ }^{17}$. Os percursos do aborto diziam respeito aos itinerários percorridos pelas jovens para ter acesso às práticas e aos métodos, tais como onde comprou o medicamento, como conseguiu a informação do local ou pessoa que vendeu e como chegou até lá. Em caso de uso de chá ou outro preparado, quem ofereceu, onde conseguiu os recursos para a compra e como tomou. As redes de apoio foram pessoas ou grupos de pessoas que auxiliaram a adolescente a realizar o aborto: familiares, companheiro, amigas, comerciantes e outros.

As entrevistas foram gravadas e transcritas em local fora dos hospitais. Para a análise, todas as entrevistas foram lidas de forma pormenorizada e, a seguir, tabuladas em um roteiro estruturado de perguntas sobre os métodos, o itinerário e as redes de apoio para abortar. O consentimento livre e esclarecido foi obtido oralmente, a fim de garantir o sigilo das narrativas. Não foi solicitada autorização dos pais ou responsáveis, e a entrevista se deu no contexto do sigilo médico. Nenhuma das participantes corria risco de vida. Nas situações em que a mãe ou outra responsável esteve presente, e após concordância ou vontade expressa da adolescente, foi também solicitado o consentimento da responsável. Os dois hospitais autorizaram a pesquisa, e o estudo foi aprovado pelo Comitê de Ética em Pesquisa do Instituto Camilo Filho.

\section{Resultados e discussão}

\section{Perfil sociodemográfico das adolescentes}

A Tabela 1 apresenta as características sociodemográficas das adolescentes. Houve uma concentração de adolescentes com menos de 18 anos, solteiras, pardas e negras, urbanas, que moravam com a família nuclear. A maioria das jovens tinha escolaridade inferior à esperada para a idade. Quase metade $(46 \%, 14)$ das adolescentes afirmaram trabalhar, sendo que $23 \%$ (7) dessas declararam ser a única fonte de renda da família. A renda familiar entre um e três salários mínimos foi a mais comum. Nenhuma delas morava sozinha e, na sua maioria $(73 \%, 22)$, elas residiam com os pais ou apenas com um deles. Doze (40\%) adolescentes tinham escolaridade adequada para a idade, o que representa a média das regiões Norte e Nordeste, mas inferior à das demais regiões do país ${ }^{18}$.

\section{Características do aborto atual}

A Tabela 2 mostra que 77\% (23) das adolescentes estavam na primeira gravidez, mas $23 \%$ (7) disseram estar na segunda gravidez, e $20 \%$ (6) dessas últimas tinham um filho vivo. A única adolescente com história de dois abortos o vivenciou no intervalo de 05 meses durante a coleta de dados da pesquisa. As mulheres que já tinham um filho afirmaram que esse fato foi importante para a decisão de abortar na segunda gravidez. Em 2012, um inquérito realizado com 122 mulheres de 18 a 39 anos que provocaram aborto investigou o perfil sociodemográfico dessas mulheres e o itinerário que elas traçaram para interromper a gravidez. Os autores verificaram que o primeiro aborto ocorreu na adolescência e que a repetição dele se deu no final dessa fase ${ }^{19}$.

A confirmação da gravidez foi feita através de teste de farmácia por 53\% (16) das adolescentes; 
$\beta$-hCG sérico por $16 \%$ (5); sintomas e sinais do atraso menstrual por 16\% (5); e ultrassonografia transvaginal por $14 \%$ (4). A Pesquisa Nacional de Aborto, na fase de entrevistas, observou dado semelhante em relação às mulheres de outras faixas etárias de cinco capitais brasileiras ${ }^{19}$. Cinco adolescentes (16\%) não realizaram teste confirmatório, sendo os sintomas e sinais do atraso menstrual suficientes para a decisão pelo aborto. A idade gestacional estimada pela última menstruação ou por ultrassonografia era de até oito semanas em $33 \%$ (10) dos casos, de nove a doze semanas em $47 \%$ (14) e superior a treze semanas em 20\% (6). A realização do aborto ainda no primeiro trimestre pode ter diminuído os riscos de complicações,

Tabela 1. Características sociodemográficas das adolescentes com aborto clandestino. Teresina, 2011.

\begin{tabular}{|c|c|c|}
\hline \multirow{2}{*}{ Características } & \multicolumn{2}{|c|}{ Adolescentes } \\
\hline & $\mathrm{N}$ & $\%{ }^{*}$ \\
\hline \multicolumn{3}{|l|}{ Idade (anos) } \\
\hline 14 a 15 & 3 & 10 \\
\hline 16 a 17 & 16 & 53 \\
\hline 18 a 19 & 11 & 37 \\
\hline \multicolumn{3}{|l|}{ Escolaridade } \\
\hline Ensino fundamental & 13 & 43 \\
\hline Ensino médio & 13 & 43 \\
\hline Ensino superior incompleto & 4 & 14 \\
\hline \multicolumn{3}{|l|}{ Cor } \\
\hline Branca & 12 & 40 \\
\hline Negra & 9 & 30 \\
\hline Parda & 9 & 30 \\
\hline \multicolumn{3}{|l|}{ Renda familiar (salário mínimo) } \\
\hline$<1$ & 2 & 7 \\
\hline 1 a 3 & 24 & 80 \\
\hline Entre 4 a 5 & 4 & 13 \\
\hline \multicolumn{3}{|l|}{ Conjugalidade } \\
\hline Solteiras & 21 & 70 \\
\hline Casadas & 9 & 30 \\
\hline \multicolumn{3}{|l|}{ Trabalho } \\
\hline Sim & 14 & 46 \\
\hline Não & 16 & 54 \\
\hline \multicolumn{3}{|l|}{ Coabitação } \\
\hline Pai e/ou mãe & 22 & 73 \\
\hline Marido & 5 & 17 \\
\hline Avó/ tia & 2 & 7 \\
\hline Patrões & 1 & 3 \\
\hline \multicolumn{3}{|l|}{ Procedência } \\
\hline Teresina & 27 & 90 \\
\hline Interior do Piauí & 2 & 7 \\
\hline Interior do Maranhão & 1 & 3 \\
\hline
\end{tabular}

*Alguns percentuais foram arredondados. pois há uma relação entre as complicações e o tempo gestacional ${ }^{20}$.

\section{Métodos, percursos \\ e redes de apoio para abortar}

A Tabela 3 apresenta os métodos adotados pelas adolescentes: o Cytotec, isoladamente, foi utilizado por $94 \%$ (28) das adolescentes, e, combinado a chás, foi usado por apenas uma adolescente para induzir o aborto. $97 \%$ (29) das adolescentes usaram de 3 a 6 comprimidos: 80\% (24) usaram por via oral e vaginal simultaneamente; $10 \%$ (3) somente por via vaginal; $7 \%$ (2) exclusivamente por via oral. A forma de uso foi em única dose por $94 \%$ (28) das adolescentes. A maioria delas $(84 \%, 25)$ recebeu o medicamento em cartela comercial com registro de Cytotec, ao passo que $13 \%$ (4) delas os receberam em papéis avulsos sem identificação da procedência. Uma única adolescente utilizou mistura de ervas abortiva (garrafada), por via oral.

Tabela 2. Distribuição das adolescentes segundo o número de gestações, forma de confirmação da gravidez, idade gestacional, duração da internação e complicações do aborto. Teresina, 2011

\begin{tabular}{lrr}
\hline & \multicolumn{2}{c}{ Adolescentes } \\
\cline { 2 - 3 } \multicolumn{1}{c}{ Variáveis } & $\mathbf{N}$ & $\%^{*}$ \\
\hline Número de gestações & & \\
$\quad$ Primeira gravidez & 23 & 77 \\
$\quad$ Segunda gravidez & 7 & 23 \\
Meio de confirmação da gravidez & & \\
Teste de farmácia & 16 & 54 \\
B-hCG & 5 & 16 \\
Sintomas e sinais & 5 & 16 \\
Ultrassonografia & 4 & 14 \\
Idade gestacional (semanas) & & \\
Até 8 & 10 & 33 \\
De 9 a 12 & 14 & 47 \\
Acima de 12 & 6 & 20 \\
Duração da internação (horas) & & \\
24 & 23 & 77 \\
72 & 2 & 7 \\
96 & 2 & 7 \\
$>$ 120 & 3 & 9 \\
Complicações & & \\
Não & 27 & 90 \\
Sim & 3 & 10 \\
Hemorragia grave & 1 & 3 \\
Perfuração uterina & 1 & 3 \\
Infecção uterina grave & 1 & 3 \\
& &
\end{tabular}


Desde o início da sua comercialização na década de 1980, o misoprostol tem sido o método predominante para abortar pelas mulheres brasileiras ${ }^{17}$. A restrição legal ao misoprostol, que culminou com a suspensão de sua venda em farmácias comuns, não impediu sua ampla utilização, mas levou as mulheres para o comércio ilegal de medicamentos. Assim, pode haver comprometimento da eficácia da droga tanto pela possibilidade de comprimido sem o princípio ativo ou com subdoses, como também pela utilização inconsistente do misoprostol pela mulher.

A Tabela 4 descreve os percursos percorridos para o aborto, revelando uma experiência bastante semelhante entre as adolescentes entrevistadas: 43\% (13) delas adquiriram sozinhas o Cytotec, e o local de aquisição mais comum foi a farmácia no bairro onde cada uma vivia (93\%, 26). As que compraram o Cytotec em farmácia, $43 \%$ o fizeram diretamente com o proprietário, a vasta maioria no próprio estabelecimento. $\mathrm{O}$ custo médio de cada comprimido foi de $\mathrm{R} \$ 26,00$, tendo variado entre $\mathrm{R} \$ 16,00$ e $\mathrm{R} \$ 60,00$. A única adolescente que fez uso de "garrafada" o adquiriu na residência da mulher especialista em ervas.

Tabela 3. Métodos, doses e vias de administração para o aborto clandestino por adolescentes. Teresina, 2011.

\begin{tabular}{lrr}
\hline & \multicolumn{2}{c}{ Adolescentes } \\
\cline { 2 - 3 } \multicolumn{1}{c}{ Variáveis } & N & $\%$ \\
\hline Método & 28 & 94 \\
$\quad$ Cytotec isolado & 1 & 3 \\
$\quad$ Cytotec + chá de boldo & 1 & 3 \\
$\quad$ Mistura de ervas ("garrafada") & & \\
Via de administração & 24 & 80 \\
$\quad$ Oral + vaginal & 3 & 10 \\
Vaginal & 3 & 10 \\
Oral & & \\
Número de comprimidos & 6 & 21 \\
3 & 16 & 55 \\
4 & 1 & 3 \\
5 & 6 & 21 \\
$\quad$ Forma de entrega & & \\
Em cartelas (com o nome & 25 & 84 \\
comercial) & & \\
Avulso (em papel de embrulho) & 4 & 13 \\
Garrafa de refrigerante (mistura & 1 & 3 \\
$\quad$ de ervas) & & \\
Número de doses & & \\
Única & & \\
Seis* & 28 & 94 \\
\hline
\end{tabular}

"incluída a adolescente que ingeriu a mistura de ervas.
A figura do vendedor do Cytotec é ainda pouco conhecida pelos estudos em saúde pública no Brasil. Um artigo analisou o enquadramento do misoprostol na imprensa brasileira e identificou indícios do perfil do vendedor: um funcionário ou ex-funcionário de farmácia, especializado em medicamentos para o corpo, tais como hormônios ou inibidores do apetite ${ }^{21}$. $\mathrm{O}$ vendedor do Cytotec para as adolescentes assumiu características semelhantes: um homem vinculado à farmácia como vendedor ou proprietário, que se encarregou de oferecer informações básicas de saúde e segurança. Uma das adolescentes con-

Tabela 4. Percursos e redes de apoio das adolescentes que fizeram aborto clandestino. Teresina. 2011.

\begin{tabular}{|c|c|c|}
\hline \multirow{2}{*}{ Características } & \multicolumn{2}{|c|}{ Adolescentes } \\
\hline & $\mathbf{N}$ & $\%$ \\
\hline \multicolumn{3}{|l|}{ Informações sobre o método } \\
\hline Amiga & 15 & 50 \\
\hline Amigo & 9 & 30 \\
\hline Namorado ou marido & 5 & 16 \\
\hline Internet & 1 & 4 \\
\hline \multicolumn{3}{|l|}{ Fonte do recurso } \\
\hline Namorado ou marido & 9 & 30 \\
\hline Recursos próprios & 9 & 30 \\
\hline Pais/irmãos & 9 & 30 \\
\hline Amigos & 3 & 10 \\
\hline \multicolumn{3}{|l|}{ Compra do medicamento } \\
\hline Própria adolescente & 13 & 43 \\
\hline Amigo & 6 & 21 \\
\hline Amiga & 2 & 7 \\
\hline Irmã/prima & 2 & 6 \\
\hline Namorado ou marido & 6 & 20 \\
\hline Patroa & 1 & 3 \\
\hline \multicolumn{3}{|l|}{ Venda do medicamento/mistura } \\
\hline \multicolumn{3}{|l|}{ Farmácia } \\
\hline Proprietário & 13 & 43 \\
\hline Balconista & 16 & 54 \\
\hline Residência (especialista em ervas) & 01 & 3 \\
\hline \multicolumn{3}{|l|}{ Motivos para ir ao hospital } \\
\hline Sangramento transvaginal & 21 & 70 \\
\hline Dor (cólicas) & 9 & 30 \\
\hline \multicolumn{3}{|l|}{ Período de latência (horas) } \\
\hline 3 a 8 & 16 & 54 \\
\hline 9 a 24 & 7 & 24 \\
\hline 25 a 72 & 4 & 13 \\
\hline$>72$ & 3 & 10 \\
\hline \multicolumn{3}{|l|}{ Acompanhante no serviço de saúde } \\
\hline Amiga & 9 & 30 \\
\hline Sozinha & 7 & 23 \\
\hline Tia & 2 & 7 \\
\hline Mãe & 12 & 40 \\
\hline
\end{tabular}


tou: Ele me disse: 'Você coloca quatro na vagina e toma dois e espera sangrar, mais ou menos umas três, quatro horas'.

O período de latência, ou o tempo que levou entre o uso do medicamento e o aparecimento de sintomas, foi de no mínimo 3 horas, tendo sido superior a 72 horas em três casos. Os sintomas, dor pélvica intensa e/ou sangramento de moderada a forte intensidade, deveriam fazer com que a adolescente procurasse o hospital. Em geral, o sangramento vaginal ou as cólicas uterinas surgiram até 24 horas após o uso do Cytotec. A adolescente que tomou a "garrafada" ingeriu uma xícara de chá, quatro vezes ao dia por três dias, começou o sangramento no terceiro dia. As adolescentes relataram que tinham conhecimento de que precisariam ir ao hospital para realizar curetagem uterina imediata ou ultrassonografia para avaliação.

Havia, no entanto, algumas regras básicas, compartilhadas por quase todas as adolescentes: elas foram instruídas a usar o medicamento à noite e em jejum, devendo procurar o hospital após seis horas de uso, mas somente em caso de sangramento vaginal intenso. A maioria das adolescentes seguiu à risca as prescrições do vendedor ou de sua rede de apoio - um cuidado estratégico para eliminar os vestígios do medicamento na vagina, segundo elas. Duas adolescentes buscaram assistência médica antes das seis horas e se justificaram pelas dores pélvicas intensas. $\mathrm{O}$ jejum assumiu conotações particulares à situação de dependência familiar das adolescentes: o estômago vazio aceleraria a curetagem, o que diminuiria o tempo de internação e, consequentemente, o afastamento injustificado da família.

A maioria $(80 \%, 24)$ das adolescentes não revelou a indução do aborto aos profissionais do atendimento hospitalar. Somente seis delas (20\%) confirmaram o uso do Cytotec, após questionamento direto do profissional responsável pela assistência. As que omitiram ou negaram o aborto justificaram-se pelo receio de sofrer discriminação, ameaça de denúncia ou exposição perante outras pessoas. Das adolescentes, $40 \%$ (12) sentiram-se vítimas de discriminação, de atitudes irônicas ou de ameaças por parte da equipe médica $\mathrm{e}$ de enfermagem, independentemente de terem revelado que haviam provocado o aborto. Uma delas resumiu seu incômodo pela cena da expulsão do feto: A enfermeira me fez olhar para o feto... ela insistiu que eu visse. Isso eu achei péssimo. A declaração dessas jovens evidencia a precariedade do acolhimento ofertado a elas nesses serviços. Outros estudos também já demonstraram que o aten- dimento nos hospitais públicos pode ser inadequado, com relatos de maus-tratos, atitudes preconceituosas e até denúncia pelos profissionais de saúde ${ }^{22,23}$. Recentemente, um inquérito realizado com 2.804 mulheres internadas por complicações do aborto em três capitais nordestinas, mostrou que 235 (8,9\% do total) delas relataram sentir-se discriminadas em decorrência da suspeita ou da certeza de terem provocado o aborto ${ }^{24}$.

Para as adolescentes, ir ao hospital seria o estágio final do ciclo do aborto, iniciado com o atraso menstrual. Muitas delas não cogitaram a possibilidade de finalizar o aborto sem assistência médica. Essa inquietação sobre o momento adequado de ir ao hospital fez com que três delas fossem internadas ainda em estágios iniciais de sangramento, o que estimulou os profissionais a tentar convencê-las a mudar de ideia: $O$ ultrassom mostrou que o feto estava vivo. Ele decidiu me internar para segurar a gravidez, embora eu não quisesse, mas ele convenceu minha mãe. A mãe $(40 \%, 12)$ e as amigas $(30 \%, 9)$ foram as acompanhantes mais comuns das adolescentes, muito embora 23\% delas tenham chegado sozinhas ao hospital. Da compra clandestina do misoprostol, passando pela espera dolorosa para apagar os vestígios do medicamento abortivo e culminando com a ida ao hospital público, muitas adolescentes descrevem esse itinerário como solitário, arriscado e incerto.

\section{Complicações do aborto}

Complicações graves foram observadas em 3 adolescentes desta pesquisa, principalmente em razão de problemas de assistência pós-aborto. A primeira ocorreu com uma adolescente de 15 anos e em primeira gravidez, procedente do interior de outro estado, com gestação de 15 semanas. $\mathrm{O}$ aborto foi provocado por ingestão oral fracionada de 6 comprimidos de Cytotec por 2 dias. Ela foi submetida a três curetagens uterinas sem anestesia, tendo ficado internada por 15 dias. Ao questionar sobre o porquê de não ser anestesiada, foi informada de que seu caso não "merecia" tal procedimento. Em estado grave, foi encaminhada para Teresina, onde foi diagnosticada perfuração uterina e infecção. Após a cirurgia, permaneceu internada por 15 dias.

Outra complicação ocorreu com uma adolescente de 15 anos, solteira, em primeira gravidez, residente no interior do Piauí, com gestação de 17 semanas. Ela havia ingerido doses fracionadas de mistura de ervas, a "garrafada" por três dias. Ao iniciar o sangramento vaginal em grande 
quantidade, procurou assistência médica local e foi transferida para Teresina por falta de condições de atendimento. Teve que viajar $1.100 \mathrm{~km}$ e mais de dez horas em busca de assistência médica, necessitou de transfusão sanguínea por choque hipovolêmico e ficou internada por cinco dias.

Por último, uma jovem de 18 anos, em segunda gravidez, com um filho de 9 meses, ainda amamentando, residente em Teresina, vivia uma gestação de 13 semanas. O aborto foi induzido com Cytotec por via vaginal e oral. Ao procurar assistência médica, foi internada para tentativa de inibir a progressão do aborto. Recebeu alta e, após dois dias, retornou com quadro de aborto infectado grave. Permaneceu internada por 15 dias para realizar curetagem uterina e usar antibióticos.

Essas complicações indicam a má prática na assistência em saúde fornecida a muitas dessas mulheres. Em outros países há evidências de que as complicações pós-aborto podem ser em parte decorrentes da qualidade do atendimento hospitalar prestado ${ }^{25}$. O risco aumentado de morte foi relacionado com o maior tempo de espera no hospital para obtenção do tratamento para complicações do aborto provocado ${ }^{26}$.

\section{Considerações finais}

A novidade deste estudo foi ter explorado um tema silenciado, porém fundamental à saúde pública no Brasil, como é o aborto entre as adolescentes. Por razões metodológicas e legais, são raros os estudos sobre a trajetória de adolescentes que abortaram clandestinamente, em particular em regiões periféricas do país. O Cytotec é o principal método para o aborto clandestino utilizado por adolescentes em Teresina. A quase totalidade das adolescentes entrevistadas fez uso de Cytotec vaginal e/ou oral para iniciar o aborto sozinha. Assim como outros estudos nacionais já mostraram, as adolescentes adquirem o Cytotec de vendedores de farmácia, uma figura central para a transmissão de cuidados básicos de saúde e instruções de uso do medicamento. Sozinhas ou acompanhadas das mães ou amigas, as adolescentes buscam os hospitais para finalizar o aborto, mas somente após uma espera média de oito horas, intervalo considerado suficiente para apagar os vestígios do medicamento na vagina.

Três adolescentes apresentaram complicações graves de saúde em decorrência do aborto clandestino. Os relatos das adolescentes indicam que as complicações não foram consequência direta do método utilizado (duas delas haviam usado o Cytotec e uma tinha ingerido "garrafada"), mas da precariedade da assistência às mulheres em processo de abortamento. A maioria das adolescentes omitiu dos médicos e enfermeiras que haviam provocado o aborto, por receio de sofrer discriminação ou maus-tratos. A combinação entre a ineficiência dos serviços e o controle da informação pelas mulheres aumenta o risco de consequências graves à saúde. As complicações vivenciadas pelas adolescentes apontam para a urgente necessidade de capacitação e sensibilização dos profissionais de saúde para o atendimento às mulheres em processo de abortamento.

\section{Colaboradores}

MD Nunes realizou as entrevistas. MD Nunes, A Madeiro e D Diniz redigiram o artigo. 


\section{Referências}

1. Gupta N, Leite IC. Adolescent fertility behavior: trends and determinants in Northeast Brazil. Int Fam Plann Perspect 1999; 25(3):125-131.

2. Senanayake P, Faulkner KM. Unplanned teenage pregnancy. Best Pract Res Clin Obstet Gynaecol 2003; 17(1):117-129.

3. Brasil. Ministério da Saúde (MS). Estudo da Mortalidade das Mulheres de 10 a 49 anos, com ênfase na mortalidade materna: relatório final. Brasília: MS; 2006.

4. Brasil. Ministério da Saúde (MS). Parto, Aborto e Puerpério: assistência humanizada à mulher. Brasília: MS; 2001.

5. Berquó E, Garcia S, Lima L. Reprodução na juventude: perfis sociodemográficos, comportamentais e reprodutivos na PNDS 2006. Rev Saude Publica 2012; 46(4):685-693.

6. Sing S. Adolescent childbearing in developing countries: a global review. Stud Fam Plann 1998; 29(2): 117-136.

7. Sedgh G, Singh S, Shah IH, Ahman E, Henshaw SK, Bankole A. Induced abortion: incidence and trends worldwide from 1995 to 2008. Lancet 2012; 379(9816):625-632.

8. Ahman E, Shah IH. New estimates and trends regarding unsafe abortion mortality. Int J Gynecol Obstet 2011; 115(2):121-126.

9. Shah IH, Ahman E. Unsafe abortion differentials in 2008 by age and developing country regions: high burden among young people. Reprod Health Matters $2012 ; 20(39) ; 169-173$

10. Diniz D, Medeiros M. Aborto no Brasil: uma pesquisa domiciliar com técnica de urna. Cien Saude Colet 2010; 15(Supl. 1):959-966.

11. Viggiano MGC, Faundes A, Viggiano ABF, Souza GR, Rebello I. Disponibilidade de misoprostol e complicações de aborto provocado em Goiânia. J Bras Ginecol 1996; 106(3):55-61.

12. Faundes A, Santos LC, Carvalho M, Gras C. Postabortion complications after interruption of preg nancy with misoprostol. Adv Contrac 1996; 12(1):1-9.

13. Menezes GMS, Aquino EML, Silva DO. Aborto provocado na juventude: desigualdades sociais no desfecho da primeira gravidez. Cad Saude Publica 2006; 22(7):1431-1446.

14. Peres SO, Heilborn ML. Cogitação e prática do aborto entre jovens em contexto de interdição legal: o avesso da gravidez na adolescência. Cad Saude Publica 2006; 22(7):1411-1420.

15. Correia DS, Cavalcante JC, Egito EST, Maia EMC. Prática do abortamento entre adolescentes: um estudo em dez escolas em Maceió (AL, Brasil). Cien Saude Colet 2011; 16(5):2469-2476.

16. Chaves JHB, Pessini L, Bezerra AFS, Rego G, Nunes R. A interrupção da gravidez na adolescência: aspectos epidemiológicos numa maternidade pública no nordeste do Brasil. Saúde Soc 2012; 21(1):246-256
17. Corrêa MCDV, Mastrella M. Aborto e misoprostol: usos médicos, práticas de saúde e controvérsia científica. Cien Saude Colet 2012; 17(7):1777-1784.

18. Instituto Brasileiro de Geografia e Estatística (IBGE). SIS 2010: mulheres mais escolarizadas são mães mais tarde e têm menos filhos [Internet] 17 set. 2010 [acessado 2012 abr 3]. Disponível em: www. ibge.gov.br/home/presidencia/noticia_visualiza. php?id_noticia+1717\&id_pagina $=1$

19. Diniz D, Medeiros M. Itinerários e métodos do aborto ilegal em cinco capitais brasileiras. Cien Saude Colet 2012; 17(7):1671-1681.

20. Nader PRA, Blandino VRP, Maciel ELN. Características de abortamentos atendidos em uma maternidade pública do Município da Serra - ES. Rev Bras Epidemiol 2007; 10(4):615-612.

21. Diniz D, Castro R. O comércio de medicamentos de gênero na mídia impressa brasileira: misoprostol e mulheres. Cad Saude Publica 2011; 27(1):94102 .

22. Bertolani GBM, Oliveira EM. Mulheres em situação de abortamento: estudo de caso. Saúde e Sociedade 2010; 19(2):286-301.

23. Diniz D, Madeiro A. Cytotec e aborto: a polícia, os vendedores e as mulheres. Cien Saude Colet 2012; 17(7):1795-1804

24. Aquino EML, Menezes G, Barreto-de-Araújo TV, Alves MT, Alves SV, Almeida MCC, Schiavo E, Lima LP, Menezes CAS, Marinho LFB, Coimbra LC, Campbell O. Qualidade da atenção ao aborto no Sistema Único de Saúde do Nordeste brasileiro: o que dizem as mulheres? Cien Saude Colet 2012; 17(7): 1765-1776.

25. Grimes DA, Benson J, Singh S, Romero M, Ganatra B, Okanofua FE, Shah IH. Unsafe abortion: the preventable pandemic. Lancet 2006; 368(9550):19081919.

26. Mayi-Tsonga S, Oksana L, Ndombi I, Diallo T, Sousa $\mathrm{MH}$, Faúndes A. Delay in the provision of adequate care to women who died from abortion-related complications in the principal maternity hospital of Gabon. Reprod Health Matters 2009; 17(34): 65 70 .

Artigo apresentado em 16/10/2012

Aprovado em 20/10/2012

Versão final apresentada em 24/10/2012 